\title{
Undiagnosed giant cystic pheochromocytoma: A case report
}

\author{
HUAN-LIANG WANG ${ }^{1}$, BAO-ZHU SUN ${ }^{1}$, ZHI-JIE XU $^{1}$, WEI-FU LEI ${ }^{1}$ and XIAO-SHUANG WANG ${ }^{2}$ \\ ${ }^{1}$ Department of Anesthesiology, Qilu Hospital, Shandong University; ${ }^{2}$ College of Pharmacy, \\ Shandong University of Traditional Chinese Medicine, Jinan, Shandong 250012, P.R. China
}

Received October 4, 2014; Accepted June 25, 2015

DOI: $10.3892 / \mathrm{ol} .2015 .3484$

\begin{abstract}
Giant cystic pheochromocytomas (GPCCs) are rare adrenal tumors and the majority of them present as asymptomatic. As a result GPCCs often remain undiagnosed until surgery and therefore the surgical team face a greater challenge in perioperative management. The present study describes the case of a 36 year-old woman with an undiagnosed GPCC, which was successfully resected despite the occurrence of perioperative cardiovascular events, including hypertension, hypotension, ventricular arrhythmias, acute heart failure, acute myocardial infarction, and the patient was discharged home without any recurrence. It should be considered in retroperitoneal tumour of patients with nonspecific symptoms and given adequate treatment to promote the perioperative safety.
\end{abstract}

\section{Introduction}

Pheochromocytoma (PCC), a rare catecholamine-producing tumor with an estimated incidence of $0.005-0.1 \%$ in the worldwide population (1), may result in classical symptoms, including severe hypertension accompanied by headache and palpitation requiring proactive preoperative medical management to decrease morbidity and mortality. However, there are certain exceptions that have been described traditionally as the ' $10 \%$ rule', as they occur at an incidence of $\sim 10 \%$ within patients with PCC $(1,2)$. 'Silent' PCC is one of the exceptions that does not exhibit classic PCC symptoms (3); therefore, 'silent' PCC often remains undiagnosed until surgical excision occurs and the anesthesia teams face a greater challenge. The authors report the case of a silent giant cystic pheochromocytoma (GPCC), which was preoperatively diagnosed as a malignant renal mass; GPCC was confirmed as a result of the classical hypertension crisis following surgical exploration and histopathological evaluation.

Correspondence to: Professor Wei-Fu Lei, Department of Anesthesiology, Qilu Hospital, Shandong University, 107 Wenhuan Xi Road, Jinan, Shandong 250012, P.R. China

E-mail: leiweifu001@163.com; timw4788@gmail.com

Key words: pheochromocytoma, renal tumor, hypertension crisis

\section{Case report}

Written informed consent was obtained from the patient and the institutional ethics review board was consulted for approval (not deemed necessary by the Institutional Ethics Review Board of Qilu Hospital, Jinan, China) for publishing this case.

A 36-year-old woman presented to Qilu Hospital of Shandong University on May 9, 2013, with the primary complaint of abdominal discomfort following eating and lumbodorsal distending pain for 3 months, and reported weight loss of $8 \mathrm{~kg}$ during this time. The patient's medical history included a caesarean section and an ovarian cysts surgery, but no history of hypertension or headache. The patient's vital signs included an arterial blood pressure of 120/80 $\mathrm{mmHg}$, heart rate of $80 \mathrm{bpm}$ and temperature $36.8^{\circ} \mathrm{C}$. The only significant finding during physical examination was for left renal region percussion pain. Laboratory analysis identified a slightly elevated blood glucose level of $7.73 \mathrm{mmol} / 1$ (normal range, 3.90-6.10 mmol/l). Ultrasonography examination revealed a cystic space-occupying lesion $(10.3 \times 9.3 \mathrm{~cm})$ in the left upper abdomen, which was considered to be a left renal cystic mass. Abdominal computed tomography (CT) and a contrast-enhanced CT scan demonstrated a giant cystic-solid mass on the left kidney, which occupied a large part of the superior abdominal cavity (Fig. 1). Based on the patient's age, gender, history, physical examination, and preoperative imaging, a clinical diagnosis of a malignant adrenal mass was suspected. No special treatment was given prior to surgery because the diagnosis of a pheochromocytoma had not been considered.

Standard monitors were applied and the blood pressure, heart rate and peripheral capillary oxygen saturation $\left(\mathrm{SpO}_{2}\right)$ measurements were $108 / 70 \mathrm{mmHg}, 80 \mathrm{bpm}$ and $97 \%$ on arrival in the operating room. General anesthesia was induced with: Midazolam, $3 \mathrm{mg}$; etomdate, $12 \mathrm{mg}$; and fentanyl, $0.2 \mathrm{mg}$. Rocuronium bromide, $50 \mathrm{mg}$, was given to facilitate the intubation of the larangeal mask airway (LMA), and anesthesia was maintained with $1.5-2.5 \%$ sevoflurane. When the mass was investigated, the anesthesiologist noted the blood pressure and heart rate rapidly increased to $>210 / 120 \mathrm{mmHg}$ and $120 \mathrm{bpm}$, respectively, and ventricular premature contractions became frequent. Investigation of the mass was immediately ceased because it became apparent that the mass may was potentially a pheochromocytoma. Intravenous (i.v.) phentolamine (1 mg injection), nitroprusside (4 mg drip), esmolol (30 mg injection) were administrated following deepening anesthesia, and a radical arterial puncture catheter was inserted to aid in 
the monitoring of arterial blood pressure. The patient experienced multiple episodes of hypertension alternating with hypotension, and this resulted in the $\mathrm{SpO}_{2}$ reducing to $89 \%$ and the $\mathrm{PaO}_{2}$ reducing to $61 \mathrm{mmHg} 40$ min later. Meanwhile, a moist rale became obvious in the bilateral lungs following auscultation of the chest. An endotracheal tube was intubated to replace the LMA. PEEP (5-10 $\left.\mathrm{cm} \mathrm{H}_{2} \mathrm{O}\right)$, dihydroxypropyl theophylline (10 g i.v. drip), dexamethasone (10 g i.v. drip), fursemide (10 g i.v. injection) were administered to inhibit the development of pulmonary edema. $5 \% \mathrm{NaHCO}_{3}, 10 \%$ potassium chloride were administrated according the results of blood gas analysis. When the blood circulation was under control, the mass was quickly excised and the blood pressure rapidly reduced to $60 / 40 \mathrm{mmHg}$. Following administration of intravenous norepinephrine and a larger volume of liquids infusion thought a central venous catheter, the blood pressure was maintained at $>100 / 60 \mathrm{mmHg}$. During the dissection, the mass was identified and appeared to originate from the left adrenal gland. The patient was transferred to the ICU with the support of a portable breathing machine for further monitoring and treatment of the hypotension at the end of surgery. Inverted $\mathrm{T}$ waves were observed in the V1-V5 chest leads of the electrocardiogram from when the patient was transferred to the intensive care unit for 7 days post-surgery. Increases in serum N-terminal pro-brain natriuretic peptide $(6,316.0 \mathrm{pg} / \mathrm{ml}$, normal value $<450 \mathrm{pg} / \mathrm{ml})$ and troponin I $(0.48 \mathrm{ng} / \mathrm{ml}$, normal value $<0.06 \mathrm{ng} / \mathrm{ml}$ ) were also observed, which indicated the occurrence of myocardial damage. Following comprehensive treatment with a continuous pump of norepinephrine $(2 \mathrm{mg}$ i.v. drip), the patient was transferred back to the surgical ward a week later. Two days later, norepinephrine treatment was stopped and the patient was discharged home on postoperative day 14 without any recurrence. During a follow-up examination performed 7 days after discharge from hospital, the patient exhibited favorable results without any discomfort. In addition, all laboratory examination and hemodynamic index results were within the normal ranges.

Gross histopathological evaluation revealed a 10x9 cm, cyst-solid mass in the adrenal gland. Formalin-fixed tissue was embedded into paraffin wax and cut into 5- $\mu \mathrm{m}$ thick sections. The sections were stained with hematoxylin and eosin for histopathological examination or incubated with specific antibodies against chromogranin A, synaptophysin, CD5 and Melan A and stained with DAB for immunohistochemical evaluation. The sections were examined and images were captured with an Olympus CX21 light microscope (Olympus Corporation, Greenhills, Philippines) at a magnification of x100. Representative histopathological images are presented in Fig. 2, which confirmed the histopathological diagnosis of PCC.

\section{Discussion}

PCCs have an estimated incidence of $<0.1 \%$ in the hypertensive population (4). They are catecholamine-secreting tumors derived from chromaffin cells originating in the neural crest and from cells of similar embryonic derivation within the sympathetic ganglia (1). PCCs are highly vascular tumors, which are commonly unilateral and solitary. However, there are certain exceptions which have been described traditionally

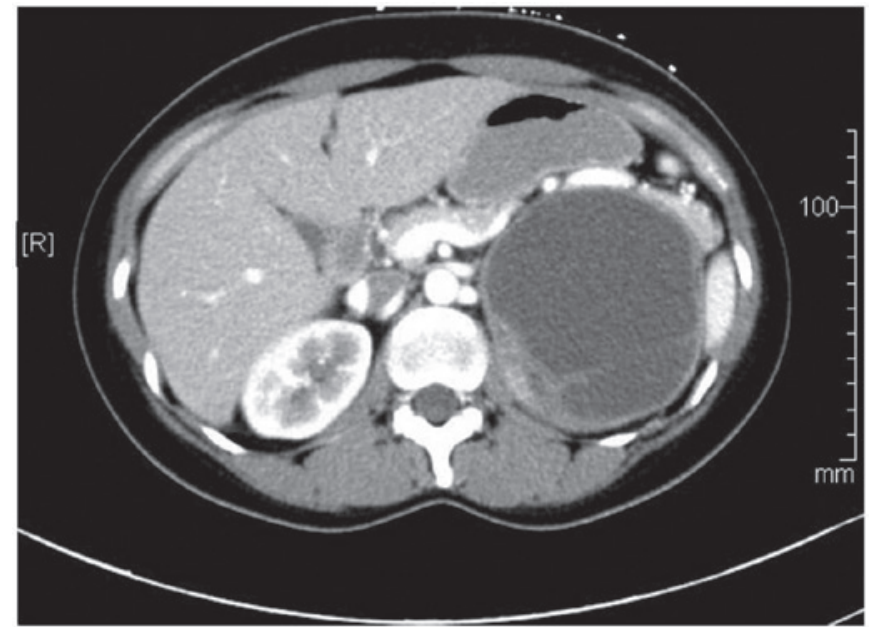

Figure 1. Contrast-enhanced computed tomography scan of the abdomen demonstrates an $11 \mathrm{~cm}$ cystic-solid mass associated with the left kidney.

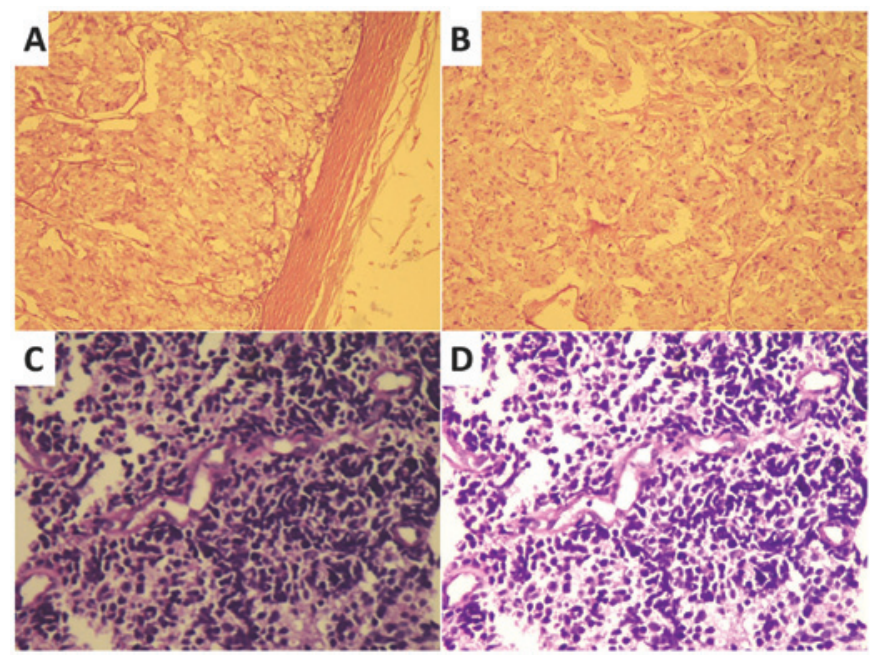

Figure 2. Typical histopathological pictures of tumor within adrenal gland. (A) The tumor has a thick fibrotic capsule without capsular invasion (hematoxylin-eosin, 10x10). (B) The neoplastic cells are characteristically arranged in well-defined nests ('Zellballen') bound by a delicate fibrovascular stroma. The cells have a finely granular cytoplasm. The nuclei are usually round or oval with prominent nucleoli. (C) Cells stained brown are positive for chromogranin A. (D) Cells stained brown are positive for synaptophysin (hematoxylin-eosin staining; magnification, x100).

as the ' $10 \%$ rule' with a $\sim 10 \%$ incidence of extra-adrenal location, familial occurrence, childhood onset, malignant phenotype, recurrence following resection, multiple or bilateral tumor formation and the absence of symptoms. The ' $10 \%$ rule' has been challenged by developments in clinical and molecular research. A previous study has indicated that as many as $25 \%$ of PCCs may be familial (5), and asymptomatic and malignant PCC occur in $>10 \%$ of PCC patients (1).

Typical PCCs are 3-5 cm in diameter, weighing 50-90 g. GPCCs weighing $>1 \mathrm{~kg}$ are rarely observed. Pan et al (6) summarized 15 cases of GPCCs from the English literature in the 30 years between 1976 and 2006: The authors observed that GPCCs occurred more frequently in males (male to female ratio, 2:1), the mean age was 45 years (ranging between 12 and 70 years), the classical symptoms are attacks of severe 
hypertension accompanied by headache and palpitation, but the majority of GPCCs were asymptomatic (9/15). The factors which contributed to the GPCCs presenting as asymptomatic included the number of cells producing catecholamine, which significantly reduced with the presence of an extensive necrotic cystic region at the centre of the mass; when interstitial tissue composes the majority of the neoplasm and the cells are thus not bioactive; and that the catecholamines and metabolic products stored in the capsular mass are not released into the blood circulation until the PCC mass is isolated (3). Thus, patients with GPCC may not manifest the typical clinical manifestations or elevated levels of urinary catecholamine metabolites. Abdominal CT scan also may not accurately delineate the organ site of mass in the case of GPCCs. Clinically, GPCCs may mimic cystic neoplasms of the pancreas (7), liver (8), pelvis (9) or kidney as in the present case report. Large adrenal tumors may also cause atrophy of the gland, making the residual gland unrecognizable. Therefore, they are often not accurately diagnosed until the time of resection, when hemodynamic instability occurs, or even following evaluation of pathological specimens (7,9-13).

Once PCC is diagnosed, surgical removal of the tumor is the preferred treatment. Hypertensive crises and arrhythmias, which are directly associated with the increase of preoperative complications or even mortality, may occur even if patients are preoperatively normotensive and asymptomatic. Therefore, blocking the effects of released catecholamines are recommended for all patients with biochemically positive PCCs (14). However, in the case of silent PCCs which are not diagnosed until the tumor is further investigated, surgeons may prefer to complete the resection immediately following achieving control over the hypertension during surgery $(3,7,10,15,16)$ compared with postponing the surgery until later (17), despite the evidence that this may result in a hypertensive crisis $(3,7)$, ventricular ectopic rhythms and cardiac arrest (10), or acute myocardial damage as in the present report. When unexpected hypertension occurs during resection of an abdominal tumor, the anesthesiologist and surgeon must consider the diagnosis of pheochromocytoma and clearly realize that it is an anesthetic challenge. Good coordination between the surgical and anesthesia teams is essential for the patient's safety during surgery (7). A surgeon may be able to carefully manipulate the tumor with early isolation of tumors' venous drainage, halting intermittently to allow the anesthesia team to make the hemodynamics stable with appropriate vasoactive drugs, this coordincation may minimize the risk of an intraoperative hypertensive crisis.

GPCCs are rare adrenal tumors and the majority of them are asymptomatic. This leads to a high proportion of these tumors being undiagnosed until surgery and surgical teams face a great challenge in perioperative management as a result. The present study reports a case of GPCC; the tumor was successfully resected despite the occurrence of perioperative cardiovascular events, and the patientwas discharged home without any recurrence.

\section{Acknowledgements}

The present study was partly supported by Shandong Provincial Natural Science Foundation, P.R. China (Y2007C115 ZR2011HM028, to Dr Huan-Liang Wang and 2009ZRB14031 to Dr Wei-Fu Lei).

\section{References}

1. Dahia PL: Evolving concepts in pheochromocytoma and paraganglioma. Curr Opin Oncol 18: 1-8, 2006.

2. Elder EE, Elder G and Larsson C: Pheochromocytoma and functional paraganglioma syndrome: No longer the $10 \%$ tumor. J Surg Oncol 89: 193-201, 2005.

3. Li C, Chen Y, Wang W and Teng L: A case of clinically silent giant right pheochromocytoma and review of literature. Can Urol Assoc J 6: E267-E269, 2012.

4. Lee TH, Slywotsky CM, Lavelle MT and Garcia RA: Cystic pheochromocytoma. Radiographics 22: 935-940, 2002.

5. Neumann HP, Berger DP, Sigmund G, Blum U, Schmidt D, Parmer RJ, Volk B and Kirste G: Pheochromocytomas, multiple endocrine neoplasia type 2 and von Hippel-Lindau disease. N Engl J Med 329: 1531-1538, 1993.

6. Pan Z, Repertinger S, Deng C and Sharma P: A giant cystic pheochromocytoma of the adrenal gland. Endocr Pathol 19:133-138, 2008.

7. Antedomenico E and Wascher RA: A case of mistaken identity: Giant cystic pheochromocytoma. Curr Surg 62: 193-198, 2005.

8. Wu JS, Ahya SN, Reploeg MD, Singer GG, Brennan DC, Howard TK and Lowell JA: Pheochromocytoma presenting as a giant cystic tumor of the liver. Surgery 128: 482-484, 2000

9. Terk MR, de Verdier H and Colletti PM: Giant extra-adrenal pheochromocytoma: Magnetic resonance imaging with gadolinium-DTPA enhancement. Magn Reson Imaging 11: 47-50, 1993.

10. Melegh Z, Rényi-Vámos F, Tanyay Z, Köves I and Orosz Z: Giant cystic pheochromocytoma located in the renal hilus. Pathol Res Pract 198: 103-106, 2002.

11. Chan FK, Choi KL, Tiu SC, Shek CC and Au Yong TK: A case of giant malignant phaeochromocytoma. Hong Kong Med J 6: $325-328,2000$

12. Grissom JR, Yamase HT and Prosser PR: Giant pheochromocytoma with sarcoidosis. South Med J 72: 1605-1607, 1979.

13. Awada SH, Grisham A and Woods SE: Large dopamine-secreting pheochromocytoma: Case report. South Med J 96: 914-917, 2003.

14. Pacak K, Eisenhofer G, Ahlman H, Bornstein SR, Gimenez-Roqueplo AP, Grossman AB, Kimura N, Mannelli M, McNicol AM and Tischler AS; International Symposium on Pheochromocytoma: Pheochromocytoma: Recommendations for clinical practice from the First international Symposium. Nat Clin Pract Endocrinol Metab 3: 92-102, 2007.

15. Basiri A and Radfar MH: Giant cystic pheochromocytoma. Urol J 7: 16, 2010.

16. Costa SR, Cabral NM, Abhrão AT, Costa RB, Silva LM and Lupinacci RA: Giant cystic malignant pheochromocytoma invading right hepatic lobe: Report on two cases. Sao Paulo Med J 126: 229-231, 2008.

17. Tarant NS, Dacanay RG, Mecklenburg BW, Birmingham SD, Lujan E and Green R: Acute appendicitis in a patient with undiagnosed pheochromocytoma. Anesth Analg 102: 642-643, 2006. 